\title{
X-ray observations of sub-mm LABOCA galaxies in the eCDFS
}

\author{
I. Georgantopoulos ${ }^{1,2}$, E. Rovilos ${ }^{3}$, and A. Comastri ${ }^{1}$
}

\author{
1 INAF-Osservatorio Astronomico di Bologna, via Ranzani 1, 40127 Bologna, Italy \\ e-mail: ioannis.georgantopoulos@oabo.inaf.it \\ 2 Institute of Astronomy \& Astrophysics, National Observatory of Athens, Palaia Penteli, 15236 Athens, Greece \\ ${ }^{3}$ Max Planck Institut für extraterrestrische Physik, Giessenbachstraße, 85748 Garching, Germany \\ Received 17 June 2010 / Accepted 15 October 2010
}

\section{ABSTRACT}

\begin{abstract}
We explore the X-ray properties of the 126 sub-mm galaxies (SMGs) of the LABOCA survey in the CDFS and the eCDFS regions. SMGs are believed to experience massive episodes of star formation. Our goal is to examine whether star formation coexists with AGN activity, determine the fraction of highly obscured AGN, and finally to obtain an idea of the dominant power mechanism in these sources. Using Spitzer and radio arcsecond positions for the SMGs, we find 14 sources with significant X-ray detections. For most of these there are only photometric redshifts available, with their median redshift at $\sim 2.3$. Taking only the CDFS area into account that has the deepest X-ray observations, we estimate an X-ray AGN fraction of $<26 \pm 9 \%$ among SMGs. The X-ray spectral properties of the majority of the X-ray AGN that are associated with SMGs are consistent with high obscuration, $>10^{23} \mathrm{~cm}^{-2}$, but there is no unambiguous evidence of Compton-thick sources. Detailed spectral energy distribution fittings show that the bulk of total IR luminosity originates in star forming processes, although a torus component is usually present. Finally, stacking analysis of the $\mathrm{X}$-ray undetected SMGs reveals a signal in the soft $(0.5-2 \mathrm{keV})$ and marginally in the hard $(2-5 \mathrm{keV}) \mathrm{X}$-ray bands. The hardness ratio of the stacked signal is relatively soft $(-0.40 \pm 0.10)$ corresponding to $\Gamma \sim 1.6$. This argues against a high fraction of Compton-thick sources among the X-ray undetected SMGs.
\end{abstract}

Key words. X-rays: general - X-rays: diffuse background - X-rays: galaxies - astrochemistry

\section{Introduction}

The advent of the SCUBA detector (Holland et al. 1999) on the James Clerk Maxwell Telescope has brought a spectacular advance in the field of submillimeter (sub-mm) cosmology. The first extragalactic surveys at $850 \mu \mathrm{m}$ revealed a population of luminous, high-redshift sub-mm galaxies or SMGs (see Blain et al. 2002 , for a review). Submillimeter surveys are very fruitful in detecting distant galaxies because of the negative K-correction at sub-mm wavelengths, which counteracts the dimming of light with increasing distance. The SMGs generate significant fractions of the energy produced by all galaxies over the history of the Universe (e.g. Smail et al. 1997; Barger et al. 1998; Hughes et al. 1998; Blain et al. 1999; Arexatga et al. 2007; Wall et al. 2008), and they are probably among the most luminous objects in the Universe. The limited spatial resolution at sub-mm wavelengths has hampered the identification of their optical counterparts. Recently, a great advance has been made as Chapman et al. $(2003,2005)$ identified a large number of SMGs through their radio counterparts and subsequently followed them with Keck spectroscopy. These studies have shown that the SMG population lies at high redshift with the median at $z=2-3$, (see also Maiolino 2008, and references therein), although the highest redshift SMGs have been found out to $z \sim 4-5$ (Capak et al. 2008; Daddi et al. 2009; Coppin et al. 2009; Wardlow et al. 2010).

Mid-infrared spectroscopy with Spitzer IRS reveals mainly star-forming spectra with implied star formation rates (SFR) as high as $1000 M_{\odot} \mathrm{yr}^{-1}$ (Valiante et al. 2007; Pope et al. 2008; Menendez-Delmestre et al. 2007, 2009). Pope et al. (2008) have obtained IRS spectra of 13 SMGs in the CDFN: 11 of these were detected with their spectra dominated by PAH features. Given the known anti-correlation between PAH emission and
AGN activity this directly suggests that star formation is the major powering mechanism in these SMGs. The median luminosity of these powerful star-forming galaxies approaches $1 \times 10^{13} L_{\odot}$ at a median redshift of $z=2.7$.

The X-ray data have identified many AGN among SMGs. Alexander et al. $(2005 \mathrm{a}, \mathrm{b})$ have found X-ray counterparts to a sample of 20 SMGs with radio counterparts from Chapman et al. (2005) in the Chandra Deep Field North (CDFN). Based on the number of X-ray detections, Alexander et al. (2005b) claim a high fraction $(75 \pm 19 \%)$ of AGN among the sub-mm galaxies that have radio counterparts. If one takes the radio undetected SMGs in the CDFN into account, making the conservative assumption that none of the radio undetected SMGs hosts AGN activity, then the AGN fraction in the SMG population becomes $>38_{-10}^{+12} \%$ (Alexander et al. 2005a). Alexander et al. (2005b) claim that the vast majority of the radio-detected SMGs are highly obscured with column densities exceeding $10^{23} \mathrm{~cm}^{-2}$. The above results suggest that the intense starformation goes hand in hand with the supermassive black-hole growth in SMGs. The low $L_{\mathrm{X}} / L_{\mathrm{FIR}}$ ratio suggests that intense star formation activity dominates the bolometric output (Alexander et al. 2005b). Laird et al. (2010) present an analysis of 35 SMGs in the CDFN with subarcsec positions either from radio or Spitzer counterparts. They find 16 objects with significant X-ray detection, or a fraction of about $45 \pm 8 \%$. However, they find that a dominant AGN contribution is required in only seven sources, or $20 \%$ of the SMG sample. This figure lies at the lower limit of previous estimates, raising questions about how common the AGN contribution in SMG is.

Recently, the LABOCA sub-mm camera on the APEX telescope has performed a sensitive sub-mm survey of the CDFS and its environs (eCDFS). The CDFS is the region of the sky with 
the deepest X-ray data available (together with the CDFN), and it therefore provides the opportunity to further explore the X-ray properties of SMGs. Lutz et al. (2010) have performed a stacking analysis in the sub-mm LABOCA map of the X-ray sources detected in the eCDFS. Their main aim is to explore the star formation properties of the X-ray AGN population as revealed by its sub-mm emission. In this paper, in a complementary study we explore the X-ray properties of the SMGs in the LABOCA survey. Our goals, analogous with the work of Alexander et al. (2005a) and Laird et al. (2010) in the CDFN, is to study the fraction of AGN among SMGs and, in particular, the fraction of heavily obscured sources and Compton-thick AGN among them. Hereafter, we adopt $H_{\mathrm{o}}=75 \mathrm{~km} \mathrm{~s}^{-1} \mathrm{Mpc}^{-1}, \Omega_{\mathrm{M}}=0.3$, and $\Omega_{\Lambda}=0.7$ throughout the paper.

\section{Data}

\subsection{The LABOCA sub-mm survey}

LABOCA is the sub-mm camera (Siringo et al. 2009) at the APEX telescope (Güsten et al. 2006). We use the $870 \mu \mathrm{m}$ map obtained by the LABOCA eCDFS sub-mm survey -LESS(Weißet al. 2009). LESS covers the full $30 \times 30$ arcmin field size of the eCDFS and has a uniform noise level of $\sigma_{870 \mu \mathrm{m}} \approx$ $1.2 \mathrm{mJy} /$ beam. This catalogue contains 126 sources detected at $>3.7 \sigma$. Simulations show that, at the above extraction limit, $95 \%$ of the sources have positional accuracy better than 8 arcsec (Weißet al. 2009).

\subsection{X-ray data}

The 2Ms CDFS observations consist of 23 pointings. The analysis of the $1 \mathrm{Ms}$ data is presented in Giacconi et al. (2002) and Alexander et al. (2003) while, the analysis of all 23 observations is presented in Luo et al. (2008). The average aim point is $\alpha=03^{\mathrm{h}} 32^{\mathrm{m}} 28.8, \delta=-27^{\circ} 48^{\prime} 23^{\prime \prime}$ (J2000). The 23 observations cover an area of $435.6 \mathrm{arcmin}^{2} .462 \mathrm{X}$-ray sources have been detected by Luo et al. (2008). The CDFS survey reaches a sensitivity limit of $1.3 \times 10^{-16} \mathrm{erg} \mathrm{cm}^{-2} \mathrm{~s}^{-1}$ in the hard $(2-8 \mathrm{keV})$ band, and $1.9 \times 10^{-17} \mathrm{erg} \mathrm{cm}^{-2} \mathrm{~s}^{-1}$ in the soft $(0.5-2 \mathrm{keV})$ band. This corresponds to a false-positive probability threshold of $10^{-6}$ (see Luo et al. 2008). The Galactic column density towards the CDFS is $0.9 \times 10^{20} \mathrm{~cm}^{-2}$ (Dickey \& Lockman 1990).

The eCDFS consists of four contiguous $250 \mathrm{ks}$ Chandra observations covering $\approx 0.3 \mathrm{deg}^{2}$ centred on the CDFS. Source detection was performed by Lehmer et al. (2005) and Virani et al. (2006). Here, we use the source detection of Lehmer et al. (2005) in which 762 sources were detected. The sensitivity limit reaches $1.1 \times 10^{-16}$ in the $0.5-2 \mathrm{keV}$ and $6.6 \times 10^{-16} \mathrm{erg} \mathrm{cm}^{-2} \mathrm{~s}^{-1}$ in the hard $2-8 \mathrm{keV}$ bands. This corresponds to a false-positive probability threshold of $10^{-6}$.

\subsection{Mid-IR}

The central regions of the CDFS were observed in the midIR by the Spitzer mission (Werner 2000) as part of the Great Observatory Origin Deep Survey (GOODS). These observations cover areas of about $10 \times 16.5 \mathrm{arcmin}^{2}$ in both fields using the IRAC $(3.6,4.5,5.8$, and $8.0 \mu \mathrm{m})$ and the MIPS $(24 \mu \mathrm{m})$ instruments onboard Spitzer. The sensitivity is $80 \mu \mathrm{Jy}(5 \sigma)$ in the $24 \mu \mathrm{m}$ MIPS band. The data products are available from the Spitzer data centre (http://data.spitzer.caltech.edu/ popular/goods/).
For the extended region bracketing the GOODS area, we used data from Spitzer's IRAC and MUSYC Public Legacy of the eCDFS (SIMPLE ${ }^{1}$, see van Dokkum et al. 2005) and the Far-Infrared Deep Extragalactic Legacy survey (FIDEL ${ }^{2}$, see Magnelli et al. 2009). SIMPLE is an IRAC survey of a $0.5 \times 0.5 \mathrm{deg}$ area centred on the CDFS. The source catalogue contains more than 60000 sources with an exposure time ranging from 2 to $4 \mathrm{~h}$ and a flux limit of $\sim 24 \operatorname{mag}(\mathrm{AB})$ in the $3.6 \mu \mathrm{m}$ band. FIDEL is a MIPS survey of the eCDFS with a median exposure time of $8000 \mathrm{~s}$ in the $24 \mu \mathrm{m}$ band and in the $30 \times 30$ arcmin area of the eCDFS. We note that the area of the eCDFS has been also observed with the Herschel far-IR mission (e.g. Shao et al. 2010), but the data are not yet publicly available.

\subsection{Optical data}

The CDFS has been imaged extensively in the optical (e.g. Giacconi et al. 2002). In this paper we make use of the CTIO4 m-MOSAIC II camera observations (Gawiser et al. 2006) taken as part of the MUSYC project. The survey is complete to a total magnitude of $R=25(\mathrm{AB})$.

\section{Sample selection}

A simple cross-correlation between the positions of the 126 LABOCA sources with those of X-ray sources from the catalogues of Luo et al. (2008) and Lehmer et al. (2005) in the CDFS and eCDFS fields, respectively, reveals 20 possible associations, using a search radius of $8^{\prime \prime}$, the same as the minimum positional accuracy of the LABOCA catalogue. To select the most reliable LABOCA - X-ray counterparts and identify any possible chance encounters, we used the mid-infrared emission from IRAC and MIPS, whose images have a much higher source density than those of LABOCA and Chandra .

We first cross-matched the positions of the LABOCA sources with the positions of the $24 \mu \mathrm{m}$ Spitzer MIPS sources. We chose this band as it is expected that there is a correlation between the sub-mm emission and that at mid-IR wavelengths (Pope et al. 2006). We used the likelihood ratio (LR) method (Sutherland \& Saunders 1992) to select the most probable counterpart, choosing the optimum likelihood ratio threshold that maximizes the sum of reliability and recovery fraction (see Luo et al. 2010; Rovilos et al. 2010). Starting with an initial search radius of $8^{\prime \prime}$ and with $L R>0.4$, we find MIPS counterparts for $87 \%$ of the LABOCA sources that fall in the area mapped by FIDEL, with a mean reliability of $70 \%$. Among the LABOCA - MIPS associations are 18/20 LABOCA sources with an X-ray counterpart. We then check the positions of the X-ray sources on the FIDEL map to look for any X-ray sources that are associated with a $24 \mu \mathrm{m}$ source, which is not the most probable counterpart to the LABOCA source. We find one such case, which we removed from our sample. We repeated the above procedure using the IRAC catalogue and map (from the SIMPLE survey) to investigate the two LABOCA - X-ray sources with no MIPS association. We find that the IRAC source associated with the X-rays is not the most probable counterpart to the LACOCA source in both cases, and removed them from our sample. We also checked the positions of the radio (VLA $-20 \mathrm{~cm}$ ) sources of Kellerman et al. (2008) at a flux limit of $43 \mathrm{mJy}(5 \sigma)$. We find radio counterparts for 5/18 LABOCA - X-ray associations (40, 57, 67, 108,

\footnotetext{
1 http://www . astro.yale.edu/dokkum/SIMPLE/

2 http://www .noao.edu/noao/fidel/
} 


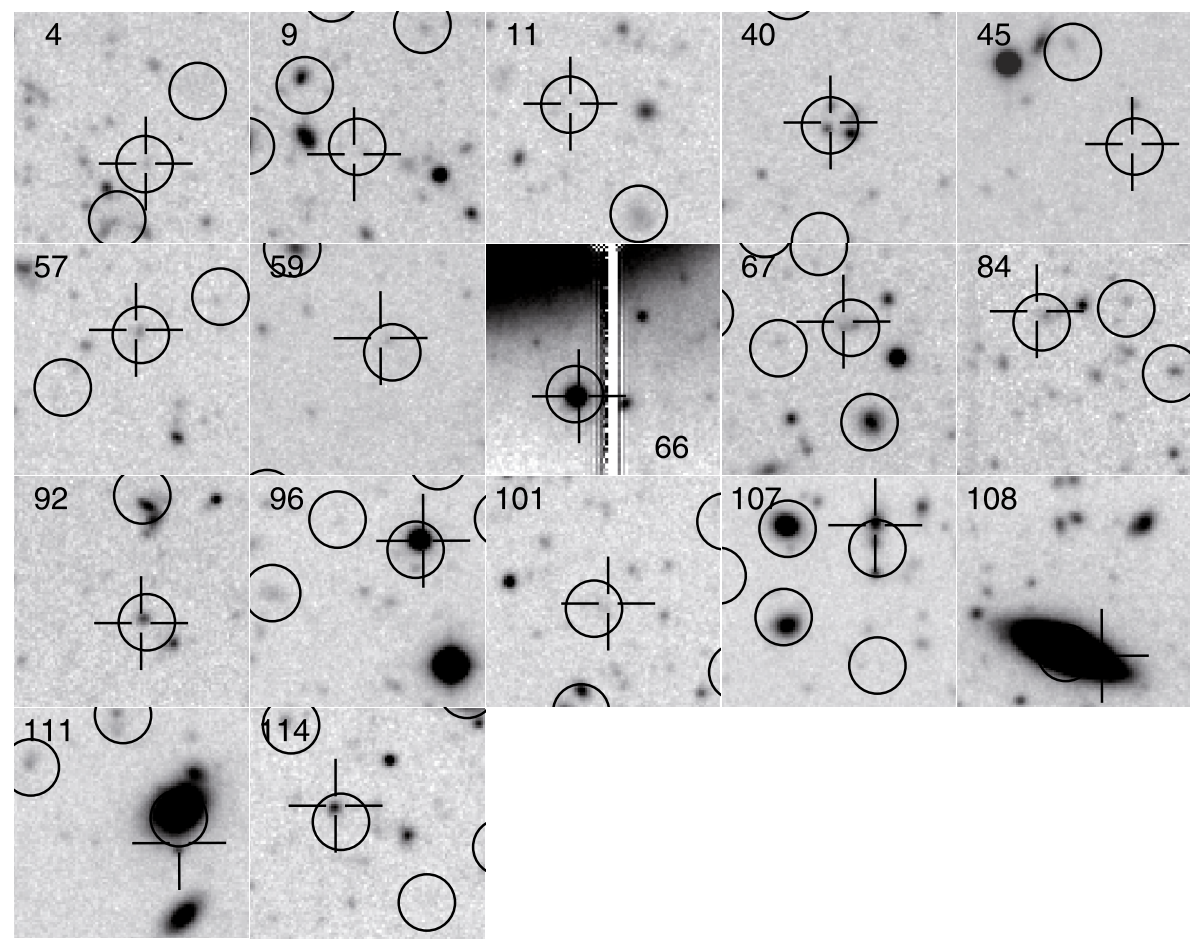

Fig. 1. The MUSYC $R$-band images. The circles ( 3 arcsec radius) show the MIPS positions. X-ray sources are denoted with crosses, preferring the CDFS $2 \mathrm{Ms}$ positions to the eCDFS, in cases where a source is detected in both surveys. The images are centred on the LABOCA positions and their size is 25 arcsec. The number at the top left of each image is the LABOCA identification.
114) and their radio positions agree with the FIDEL positions within $1.4^{\prime \prime}$

The final step is to check the optical images. In Fig. 1 we plot the MUSYC images of the 17 LABOCA - X-ray candidates. We can clearly see that there are two cases where the X-ray source is more likely to be associated with a different optical source than the one producing the bulk of the $24 \mu \mathrm{m}$ flux: W-107 and $\mathrm{W}-111$. For $\mathrm{W}-107$, the $24 \mu \mathrm{m}$ flux is coming from three discrete optical sources and the X-ray source is associated with the one that lies farther away from the LABOCA position. Therefore it is highly unlikely that the X-rays and the sub-mm emissions are physically connected. In the case of W-111, the X-ray source is different from the $24 \mu \mathrm{m}$ source, and again the X-ray and the sub-mm emissions are not associated. We removed both sources from our sample.

The X-ray counterpart of one source (W-96) is flagged as a star in Taylor et al. (2009). We checked its optical morphology using HST imaging from the GEMS survey (Rix et al. 2004) and confirm that it has an unresolved morphology with a PSF of $\sim 0.08^{\prime \prime}$ (diffraction limit in the $z_{850}$ band). The X-ray-to-optical flux ratio is low $\left(\log \left[f_{0.5-2 \mathrm{kev}} / f_{R}\right]=-1.8\right)$, confirming the stellar classification (see e.g. Rovilos et al. 2009). On these grounds we removed this source from the final sample. This of course does not suggest that this is a star with sub-mm emission, but simply that the X-rays are related to a stellar source, while there is no association of the sub-mm source with an X-ray extragalactic source.

Our final sample consists of 14 sources. Ten of them are in the region observed by the $2 \mathrm{Ms}$ CDFS, and four are in the shallower eCDFS region. The mean reliability of the sub-mm MIPS associations for these 14 sources is $87.5 \%$; i.e., we expect 1.7 spurious sources in our sample.

\section{Photometry and redshifts}

For the photometry in the optical bands, we used the MYSYC catalogues of Taylor et al. (2009) and Gawiser et al. (2006). Taylor et al. (2009) provide photometry of $\sim 17000$ sources of the eCDFS detected in the (CTIO-4 m - ISPI) $K$-band in the $U-B-V-R-I-z^{\prime}-J-H-K$ bands, and Gawiser et al. (2006) used the same optical data to detect sources in the combined $B-V-R$ band and provide photometry in the $U-B-V-R-I-z^{\prime}$ bands.

The Spitzer photometry comes from the SIMPLE and FIDEL surveys. The aperture fluxes are given in the public SIMPLE catalogue and we used the $2^{\prime \prime}$ apertures and standard aperture corrections derived from the IRAC cookbook ${ }^{3}$ to calculate the total fluxes. In the FIDEL case we used the corrected isophotal fluxes derived from the SEXTRACTOR source detection code (Bertin \& Arnouts 1996). We identify an accumulation of 6 pixels with flux higher than 1.5 times the local rms as a source. We calibrated the derived fluxes using the total fluxes of common sources between the FIDEL and MIPS-GOODS surveys. We also visually inspected the apertures derived from SEXTRACTOR to check for source blending problems, and also to check that they include all the flux of the source, since the MIPS PSF has prominent sidelobe features. We only find one such case (W-108), where the aperture used does not encompass all the flux, and we consider the measured flux as a lower limit.

Three sources in our sample (W-40, W-45, and W-67) fall in the area of GOODS that has a deeper coverage with IRAC and MIPS, as well as the HST. For these sources we used the photometry of Grazian et al. (2006), which includes optical and IRAC bands. These three, along with sources 9 and 11 fall in the MIPS-GOODS area with deeper coverage than FIDEL, so we used the GOODS $24 \mu \mathrm{m}$ photometry.

As a final step, we inspected the images of the 14 LABOCA - X-ray sources in all the bands used and compared them with with those with the smallest PSF: the optical GEMS images and (where available) the GOODS-HST images. We did that in order to detect whether the flux measured in the bands with the larger PSFs are blended with nearby sources. We find that sources W59 and W-67 are in fact double sources blended in the IRAC and

\footnotetext{
3 http://ssc.spitzer.caltech.edu/irac/ iracinstrumenthandbook/
} 
MIPS images because of limited spatial resolution, whereas for sources 9, 40, and 84, there are nearby sources that affect the MIPS flux. Source 67 is in the GOODS area and has optical and IRAC photometry from the GOODS-MUSYC survey Grazian et al. (2006), which uses PSF matching to the HST images to measure the fluxes in the optical and infrared bands, so we consider the problem to be confined only in the MIPS photometry (taken from GOODS).

Four sources (W-57, W-67, W-108, and W-114) have spectroscopic redshifts assigned to their optical, infrared, or radio counterparts: Szokoly et al. (2004), Kriek et al. (2006), Norris et al. (2006), Chapin et al. (2010) respectively. The photometric redshifts in the area of the CDFS come from Luo et al. (2010), where photometric redshifts are calculated for X-ray sources using up to $42 \mathrm{UV}$, optical, and infrared bands. The photometric redshifts in the area of the eCDFS come from Taylor et al. (2009). Finally, the photometric redshift of source W-4 in the eCDFS is calculated with the EAZY (Brammer et al. 2008) code using the $B, V, R, I$, and $z^{\prime}$ bands. This source does not have a photometric redshift in the K-selected sample of Taylor et al. (2009), as it has not been detected in this band. In Table 1 we present the X-ray, optical, and infrared fluxes, as well as the available redshifts of the sources in our sample.

\section{X-ray properties}

The X-ray properties can give a first idea of the nature of the X-ray detected SMGs. X-ray luminosities higher than about a few times $10^{42} \mathrm{erg} \mathrm{s}^{-1}$ are usually attributed to AGN activity. This limit has been dictated by the highest X-ray luminosity observed in local star-forming galaxies (e.g. Zezas et al. 1998; Moran et al. 1999). However, given that SMGs are probably the most powerful star-forming systems, it is likely that their $\mathrm{X}$-ray luminosity may surpass this limit. Star-forming systems present very little, if any, obscuration (e.g. Georgakakis et al. 2006, 2007; Tzanavaris et al. 2006; Rovilos et al. 2009, and references therein), therefore the detection of a substantial obscuring column, through X-ray spectroscopy in a low X-ray luminosity source, can further differentiate between a star-forming galaxy and an AGN.

\subsection{X-ray spectroscopy}

We used the SPECEXTRACT script in the CIAO v4.2 software package to extract the spectra of the $14 \mathrm{X}$-ray sources in our sample. The extraction radius varies between 2 and 4 arcsec with increasing off-axis angle. At low off-axis angles $(<4$ arcmin) this encircles $90 \%$ of the light at an energy of $1.5 \mathrm{keV}$. The same script extracts response and auxiliary files. The addition of the spectral, response, and auxiliary files was performed with the FTOOL tasks MATHPHA, ADDRMF and ADDARF respectively. We used the C-statistic technique (Cash 1979) specifically developed to extract spectral information from data with low signalto-noise ratio. We used the XSPEC v12.5 software package for the spectral fits (Arnaud 1996).

We fit the data using a power law absorbed by a cold absorber. We treat both the intrinsic column density and the photon index $(\Gamma)$ as free parameters. However, the photon statistics are low, in five cases not allowing us to derive meaningful constraints for both $N_{\mathrm{H}}$ and $\Gamma$. In these cases we can only fix the power-law photon index to $\Gamma=1.8$ (Nandra \& Pounds 1994; Tozzi et al. 2006), leaving only the column density $N_{\mathrm{H}}^{\text {eff }}$ as the free parameter and vice versa i.e. setting $N_{\mathrm{H}}=0$ and leaving the photon index $\Gamma^{\mathrm{eff}}$ as a free parameter. The spectral-fit results are

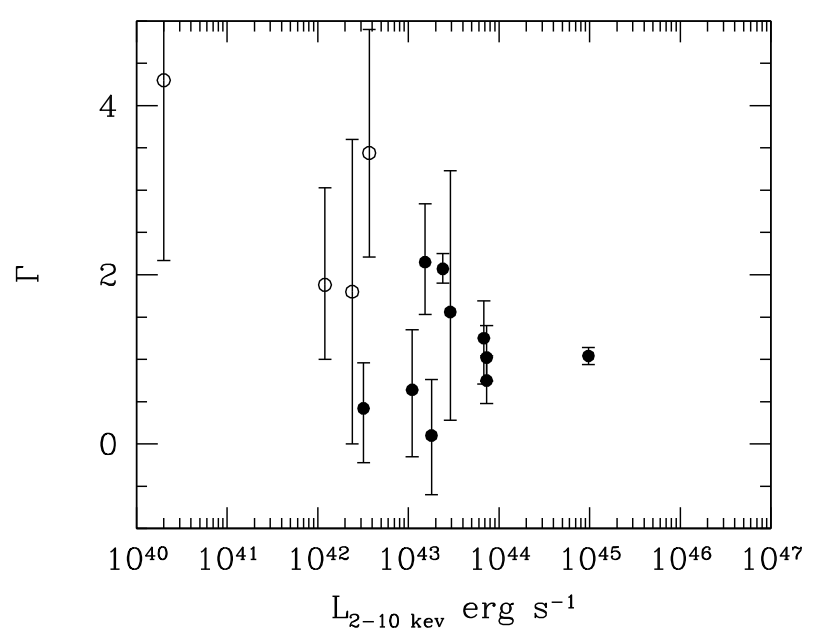

Fig. 2. The effective photon index as a function of X-ray luminosity. Filled and open circles correspond to AGN and galaxies, respectively (see text).

presented in Table 2. As is customary in X-ray astronomy, errors correspond to the $90 \%$ confidence level.

\subsection{AGN vs. star-forming galaxies}

In Fig. 2 we plot the effective photon index as a function of the obscured X-ray luminosity, i.e. Col. (7) vs. Col. (8) in Table 2. We see that nine sources have high X-ray luminosities $\left(>10^{43} \mathrm{erg} \mathrm{s}^{-1}\right)$, and thus can be considered as bona-fide AGN. Four out of the remaining low-luminosity sources present soft spectra and are thus possibly associated with star-forming galaxies. The remaining source (W-114) has a fairly hardish spectrum and so could be a highly obscured AGN: its spectrum is marginally intrinsically flat $(\Gamma<1.32$ at the $90 \%$ confidence level) and thus possibly associated with a Compton-thick AGN with a reflection-dominated spectrum.

\subsection{X-ray obscuration}

In Fig. 3 we plot the distribution of the rest-frame column densities (Col. 6 in Table 2). It is evident that most AGN (7/10) present column densities exceeding $10^{23} \mathrm{~cm}^{-2}$; i.e., they are characterised as heavily obscured AGN. One source could be a transmission dominated Compton-thick AGN (W-11). However, the uncertainty in the photometric redshift certainly makes this classification dubious.

\section{Mid-IR properties}

\subsection{Mid-IR spectral energy distributions}

We constructed the spectral energy distribution (SED) to obtain an idea on the dominant powering mechanism (AGN or star formation) in the mid-IR part of the spectrum. The SEDs are also used in order to obtain an accurate estimate of the infrared luminosities. Power-law SEDs in the mid-IR are characteristic of AGN emission (e.g. Alonso-Herrero et al. 2006; Polletta et al. 2007). A prototype power-law mid-IR SED in the local Universe is that of Mkn231, (Armus et al. 2007). This is a broad-absorption-line QSO (Braito et al. 2004) and a ultraluminous Infrared IRAS galaxy (ULIRG). On the other hand, starforming sources have a distinct dip in their mid-IR spectra at a rest-frame wavelength below $10 \mu \mathrm{m}$. Such a source is Arp 220 
Table 1. Photometry.

\begin{tabular}{|c|c|c|c|c|c|c|c|c|c|c|}
\hline $\begin{array}{l}\text { ID } \\
(1)\end{array}$ & $\begin{array}{c}\alpha \\
(2)\end{array}$ & $\begin{array}{c}\delta \\
(2)\end{array}$ & $\begin{array}{c}f_{0.5-10 \mathrm{keV}} \\
\text { (3) }\end{array}$ & $\begin{array}{c}R \\
(4)\end{array}$ & $\begin{array}{c}f_{3.6 \mu \mathrm{m}} \\
(5)\end{array}$ & $\begin{array}{l}f_{24 \mu \mathrm{m}} \\
\text { (6) }\end{array}$ & $\begin{array}{c}f_{850 \mu \mathrm{m}} \\
(7)\end{array}$ & $\begin{array}{l}L R \\
(8)\end{array}$ & $\begin{array}{c}z \\
(9)\end{array}$ & $\begin{array}{l}\text { Ref. } \\
\text { (10) }\end{array}$ \\
\hline $\mathrm{W}-4$ & 52.8996 & -27.9121 & 1.85 & 24.94 & $7.00 \pm 0.10$ & $139 \pm 5$ & $11.0 \pm 1.2$ & 5.00 & $2.58 \pm 0.9$ & 1 \\
\hline W-9 & 53.0475 & -27.8704 & 7.14 & 26.02 & $9.83 \pm 0.05$ & $122 \pm 5^{b}$ & $9.4 \pm 1.2$ & 6.92 & $3.99 \pm 0.08$ & 2 \\
\hline $\mathrm{W}-11$ & 53.0577 & -27.9334 & 1.33 & 25.61 & $6.83 \pm 0.07$ & $113 \pm 6$ & $9.2 \pm 1.2$ & 5.06 & $6.07 \pm 1.1$ & 2 \\
\hline $\mathrm{W}-40$ & 53.1950 & -27.8557 & 0.44 & 24.35 & $8.51 \pm 0.15$ & $140 \pm 4^{b}$ & $6.4 \pm 1.2$ & 6.08 & $1.90 \pm 0.02$ & 2 \\
\hline $\mathrm{W}-45$ & 53.1052 & -27.8752 & 0.06 & 27.41 & $7.50 \pm 0.27$ & $141 \pm 4$ & $6.3 \pm 1.2$ & 1.50 & $2.50 \pm 0.2$ & 2 \\
\hline W-57 & 52.9665 & -27.8908 & 1.51 & 24.33 & $7.60 \pm 0.10$ & $273 \pm 6$ & $6.1 \pm 1.3$ & 12.81 & 2.940 & 3 \\
\hline W-59 & 53.2658 & -27.7362 & 1.35 & 25.62 & $30.28 \pm 0.10^{a}$ & $453 \pm 5^{a}$ & $6.0 \pm 1.3$ & 7.67 & $1.38 \pm 0.02$ & 2 \\
\hline W-66 & 53.3830 & -27.9028 & 36.1 & 20.72 & $56.34 \pm 0.13$ & $679 \pm 9.4$ & $6.1 \pm 1.3$ & 5.37 & $0.58 \pm 0.03$ & 5 \\
\hline W-67 & 53.1803 & -27.9206 & 0.29 & 24.20 & $21.7 \pm 0.9$ & $554 \pm 4^{a}$ & $5.9 \pm 1.3$ & 15.77 & 2.122 & 4 \\
\hline W-84 & 52.9773 & -27.8513 & 1.58 & 24.97 & $12.98 \pm 0.09$ & $231 \pm 4^{b}$ & $5.5 \pm 1.3$ & 4.16 & $3.2 \pm 0.06$ & 2 \\
\hline W-92 & 52.9096 & -27.7277 & 2.98 & 24.49 & $26.57 \pm 0.10$ & $80 \pm 5$ & $5.2 \pm 1.2$ & 2.69 & $2.10 \pm 0.04$ & 5 \\
\hline W-101 & 52.9643 & -27.7649 & 1.16 & 23.01 & $5.33 \pm 0.10$ & $34 \pm 2.9$ & $5.1 \pm 1.3$ & 0.66 & $2.53 \pm 0.12$ & 2 \\
\hline W-108 & 53.3175 & -27.8445 & 1.75 & 17.36 & $516.74 \pm 0.10$ & $4040 \pm 100^{c}$ & $5.0 \pm 1.2$ & 7.89 & 0.0875 & 6 \\
\hline W-114 & 52.9628 & -27.7436 & 3.11 & 23.88 & $51.63 \pm 0.10$ & $660 \pm 9$ & $4.9 \pm 1.3$ & 2.85 & 1.605 & 7 \\
\hline
\end{tabular}

Notes. The columns are: (1) LABOCA source number in Weiß et al. (2009); (2) X-ray counterpart equatorial coordinates; (3) Total band X-ray flux in units of $\times 10^{-15} \mathrm{erg} \mathrm{s}^{-1} \mathrm{~cm}^{-2}$; (4) $R$-band magnitude (AB); (5) $3.6 \mu \mathrm{m}$ flux in units of $\mu \mathrm{Jy}$; (6) $24 \mu \mathrm{m}$ flux in units of $\mu \mathrm{Jy}$. ${ }^{(a)}$ Double-source blended in the IRAC and/or MIPS PSFs; ${ }^{(b)}$ Nearby sources possibly affecting the measured flux. ${ }^{(c)}$ Lower limit (7) LABOCA $870 \mu \mathrm{m}$ flux in units of mJy. (8) Likelihood-ratio between LABOCA and MIPS counterpart. (9) Redshift: Three and two decimal digits denote a spectroscopic and a photometric redshift respectively (10).

References. 1) EAZY; 2) Luo et al. (2010); 3) Szokoly et al. (2004); 4) Kriek et al. (2006); 5) Taylor et al. (2009); 6) Norris et al. (2006); 7) Chapin et al. (2010).

Table 2. X-ray spectral fits.

\begin{tabular}{lccccccc}
\hline \hline $\mathrm{ID}$ & $\mathrm{XID}$ & $z$ & $N_{\mathrm{H}}$ & $\Gamma$ & $N_{\mathrm{H}}(\Gamma=1.8)$ & $\Gamma\left(N_{\mathrm{H}}=0\right)$ & $L_{\mathrm{X}}$ \\
$(1)$ & $(2)$ & $(3)$ & $(4)$ & $(5)$ & $(6)$ & $(7)$ & $(8)$ \\
\hline $\mathrm{W}-4$ & LE-97 & 2.58 & $<15.3$ & $1.55_{-1.24}^{+2.14}$ & $<15.0$ & $1.56_{-1.28}^{+1.67}$ & 2.9 \\
$\mathrm{~W}-9$ & LU-114 LE-319 & 3.99 & $28.3_{-7.8}^{+8.5}$ & $1.72_{-0.19}^{+0.16}$ & $31.1_{-4.2}^{+4.2}$ & $1.04_{-0.10}^{+0.10}$ & 97. \\
$\mathrm{~W}-11$ & LU-131, LE-332 & 6.07 & $196_{-93}^{+139}$ & $2.32_{-0.67}^{+0.47}$ & $171_{-74}^{+115}$ & $1.02_{-0.28}^{+0.38}$ & 7.3 \\
$\mathrm{~W}-40$ & LU-385 & 1.90 & - & - & $21_{-13.6}^{+14.8}$ & $0.64_{-0.79}^{+0.71}$ & 1.1 \\
$\mathrm{~W}-45 \dagger$ & LU-230 & 2.50 & - & - & $<5.53$ & $3.44_{-1.23}^{+1.46}$ & 0.37 \\
$\mathrm{~W}-57$ & LU-26, LE-203 & 2.940 & $39.4_{-26.5}^{+32.9}$ & $2.60_{-1.01}^{+1.18}$ & $20.1_{-12.9}^{+15.9}$ & $1.25_{-0.54}^{+0.44}$ & 6.8 \\
$\mathrm{~W}-59 \dagger$ & LU-441 & 1.38 & - & - & $<6.1$ & $1.88_{-0.88}^{+1.15}$ & 0.12 \\
$\mathrm{~W}-66$ & LE-725 & 0.58 & $<0.17$ & $2.04_{-0.17}^{+0.25}$ & $<0.06$ & $2.07_{-0.17}^{+0.18}$ & 2.4 \\
$\mathrm{~W}-67 \dagger$ & LU-362 & 2.122 & - & - & - & 1.8 & 0.24 \\
$\mathrm{~W}-84$ & LU-33 & 3.20 & $<30.5$ & $1.03_{-0.49}^{+0.68}$ & $26.9_{-7.6}^{+16.1}$ & $0.75_{-0.27}^{+0.29}$ & 7.3 \\
$\mathrm{~W}-92$ & LE-112 & 2.10 & $3.6_{-13.4}^{+21.0}$ & $0.33_{-0.83}^{+1.53}$ & $34.7_{-18.0}^{+25.1}$ & $0.10_{-0.70}^{+0.66}$ & 1.80 \\
$\mathrm{~W}-101$ & LU-25 & 2.53 & $<7.9$ & $2.11_{-0.6}^{+1.4}$ & $<2.8$ & $2.15_{-0.62}^{+0.69}$ & 1.53 \\
$\mathrm{~W}-108 \dagger$ & LE-634 & 0.0875 & - & - & $<0.62$ & $4.3_{-2.13}^{+2.63}$ & $2 \times 10^{-3}$ \\
$\mathrm{~W}-114$ & LU-23 & 1.605 & $<45.0$ & $-0.15_{-1.02}^{+1.47}$ & $31.9_{-12.6}^{+34.2}$ & $-0.42_{-0.64}^{+0.54}$ & 0.43 \\
\hline
\end{tabular}

Notes. The columns are: (1) Laboca ID in Weiß et al. (2009); (2) CDFS (LU) or eCDFS(LE) ID in Luo et al. (2008) or Lehmer et al. (2005), respectively; (3) redshift; (4), (5) rest-frame column density and photon index in the case of sources with good photon statistics (see text); (6) restframe column density in units of $10^{22} \mathrm{~cm}^{-2}$ for fixed $\Gamma=1.8$; (7) photon-index $\Gamma$ for column density $N_{\mathrm{H}}=0$; (8) Intrinsic X-ray luminosity in the $2-10 \mathrm{keV}$ band in units of $10^{43} \mathrm{erg} \mathrm{s}^{-1}$, obtained by setting $N_{\mathrm{H}}=0$ in Col. (6); (†) Denotes a possible star-forming galaxies on the basis of the $\mathrm{X}$-ray properties i.e. low X-ray luminosity and soft spectrum (see text).

(e.g. Iwasawa et al. 2005), a ULIRG whose far-IR SED is dominated by a very strong star-forming component (Armus et al. 2007). We present the IRAC/MIPS SEDs in Fig. 4. There are only two clearcut power-law SEDs suggestive of AGN: W-4 and W-57.

Apart from this first-order approach, we fit a star-forming in combination with a torus template to the SED from $3.6 \mu \mathrm{m}$ up to $870 \mu \mathrm{m}$. We used the SED templates of Polletta et al. (2007) to produce templates combining star formation and AGN activity. As star-forming templates we used those of M 82 and Arp 220. These are two actively star-forming galaxies differing in their SFR and the amount of dust. Arp 220 is producing stars with a rate of $270 M_{\odot} \mathrm{yr}^{-1}$ (Shioya et al. 2001), and its interstellar dust gives it an infrared luminosity $>10^{12} L_{\odot}$, making it the prototypical ULIRG. M 82, on the other hand, has a more modest SFR of $10 M_{\odot} \mathrm{yr}^{-1}$ and lower mid-to-far infrared luminosity. The two SEDs are almost identical in the near-to-mid infrared and differ significantly in their mid infrared PAH and silicate features and the mid-to-far infrared dust emission. As an AGN-only SED, we used the Torus SED of Poletta et al. (2007), which is a fit to the SED of a heavily obscured type-2 QSO, SWIRE J104409.95+585224.8 (Polletta et al. 2006). We 


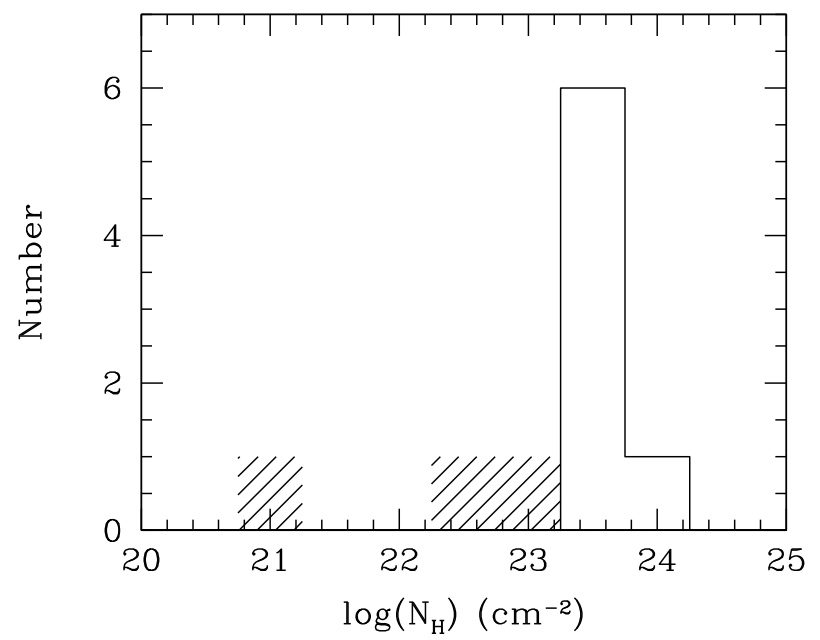

Fig. 3. The rest-frame column density distribution for the ten AGN (see text for details). The open and hatched histograms correspond to the detections and upper limits respectively.

started the SED fitting by choosing the best linear combinations of Arp 220+Torus and M 82+Torus templates using the four IRAC and the $24 \mu \mathrm{m}$ datapoints and minimizing the $\chi^{2}$ value of the fit. Then we calculated the $\chi^{2}$ values of the optimum combinations using the previous datapoints plus the sub-mm flux and chose the combination (Arp 220+Torus or M 82+Torus) that minimizes this value. In other words, we used the mid-infrared to determine the relative AGN and starburst contribution (where the two starburst templates are almost identical) and define the optimum starburst template by its prediction of the far-infrared flux. Then, we used the F-test to determine whether the AGN contribution is needed to fit the mid-infrared flux; we calculate

$F=\frac{\frac{\chi_{1}^{2}-\chi_{2}^{2}}{p_{2}-p_{1}}}{\frac{\chi_{2}^{2}}{n-p_{2}}}$

where $\chi_{1}^{2}$ and $\chi_{2}^{2}$ are the best-fit $\chi^{2}$ values of the starburst-only and combination templates, respectively, $p_{1}$ and $p_{2}$ are the degrees of freedom of the two cases (here $p_{1}=1$ and $p_{2}=2$ ), and $n$ is the points used to make the fit (here $n=5$ ). We assumed that the AGN contribution is needed if the false-rejection probability is lower than $10 \%(F>8.5)$.

The template fits are presented in Fig. 5, while the derived IR luminosities are given in Table 3 . We note that the IR-luminosity is estimated from the mid-infrared SED and the $870 \mu \mathrm{m}$ flux. This does not probe the peak of the infrared SED (around rest-frame $70 \mu \mathrm{m}$ in $v F_{v}$ ) and thus introduces an appreciable uncertainty in the determination of the far-IR luminosity. Measurements of the infrared SED at its peak (for example, with Herschel) are needed to provide accurate bolometric luminosities. However, the $6 \mu \mathrm{m}$ luminosities are hardly affected by the lack of far-IR measurements. We see that a torus component is required in most cases (eight out of ten AGN). However, the contribution of the torus to the total IR luminosity is small (see Table 3). The contribution of the torus is appreciable only in the case of the two power-law IRAC AGN (W-4 and W-57) and also in the case of the source $\mathrm{W}-11$ (reaching up to $\sim 20 \%$ ). For most candidate galaxies the bulk of the $6 \mu \mathrm{m}$ emission comes from star-forming processes. Only the candidate galaxy W-45 has a non-negligible torus component $(\sim 10 \%)$.

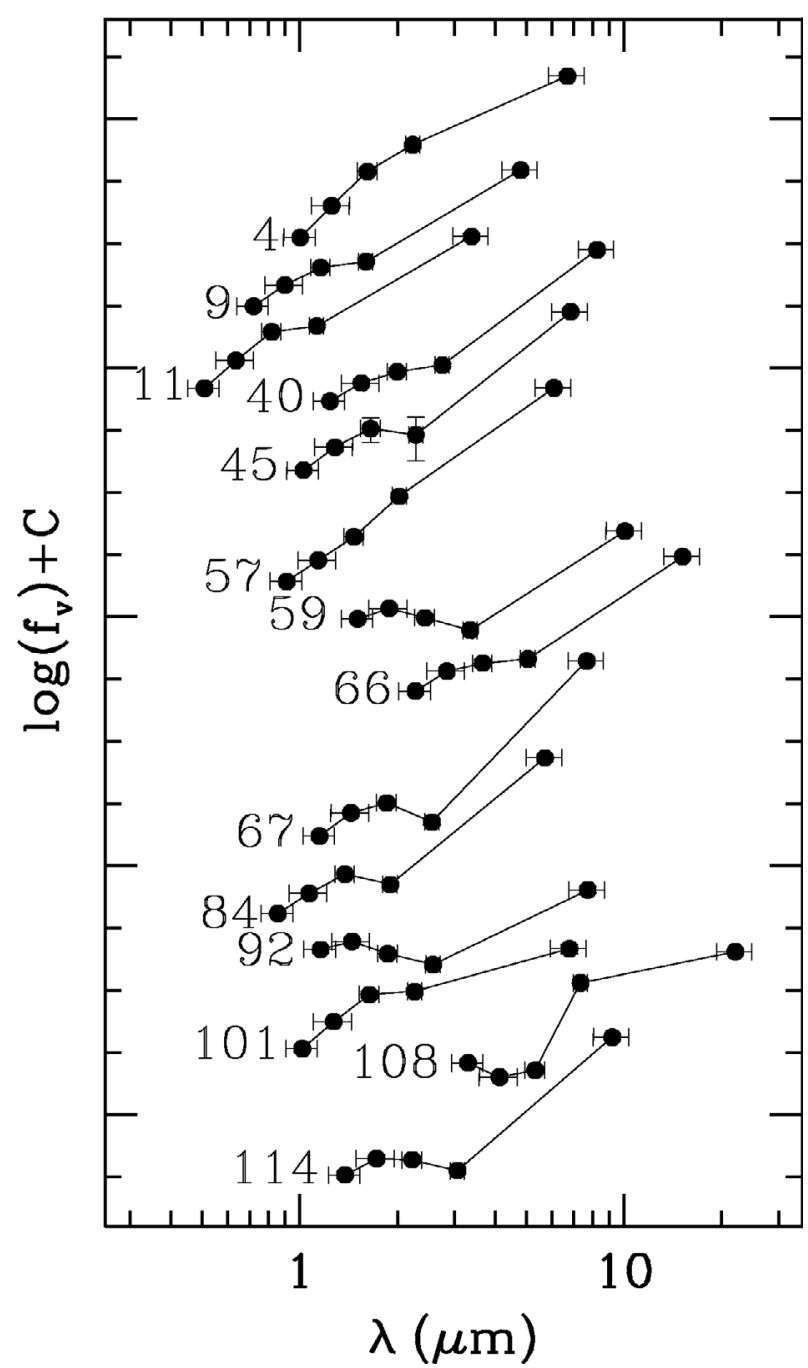

Fig. 4. The IRAC, MIPS $(24 \mu \mathrm{m})$ spectral energy distributions.

\subsubsection{X-ray to $6 \mu \mathrm{m}$ luminosity}

The derivation of the $L_{\mathrm{x}}$ to $L_{6 \mu \mathrm{m}}$ ratio has as a goal to identify any highly obscured, Compton-thick AGN. These are difficult to identify with X-ray spectroscopy especially in the case of limited photon statistics. One of the most reliable proxies of the intrinsic power of an AGN is the mid-IR monochromatic $6 \mu \mathrm{m}$ luminosity (e.g. Lutz et al. 2004). This wavelength region is more representative of the hot dust heated by the AGN and thus provides a reliable diagnostic of the AGN power (e.g. Alexander et al. 2008).

We present the $2-10 \mathrm{keV}$ absorbed luminosity against the monochromatic $6 \mu \mathrm{m}$ IR luminosity coming from the torus in Fig. 6. The area between the solid lines denotes the region of the X-ray to $6 \mu \mathrm{m}$ luminosity plane where local AGN reside (Lutz et al. 2004; Alexander et al. 2008; Bauer et al. 2010). The area below the dashed line corresponds to low X-ray luminosity sources, i.e. Compton-thick sources (or alternatively normal galaxies). We see that some sources fall in the low part of the $L_{\mathrm{x}} / L_{6 \mu \mathrm{m}}$ diagram (e.g. W-11, W-57, W-114). All four candidate galaxies, i.e. the sources with low X-ray luminosities and soft $\mathrm{X}$-ray spectra fall, as well, in the lower part of this diagram. 
I. Georgantopoulos et al.: X-ray observations of sub-mm galaxies
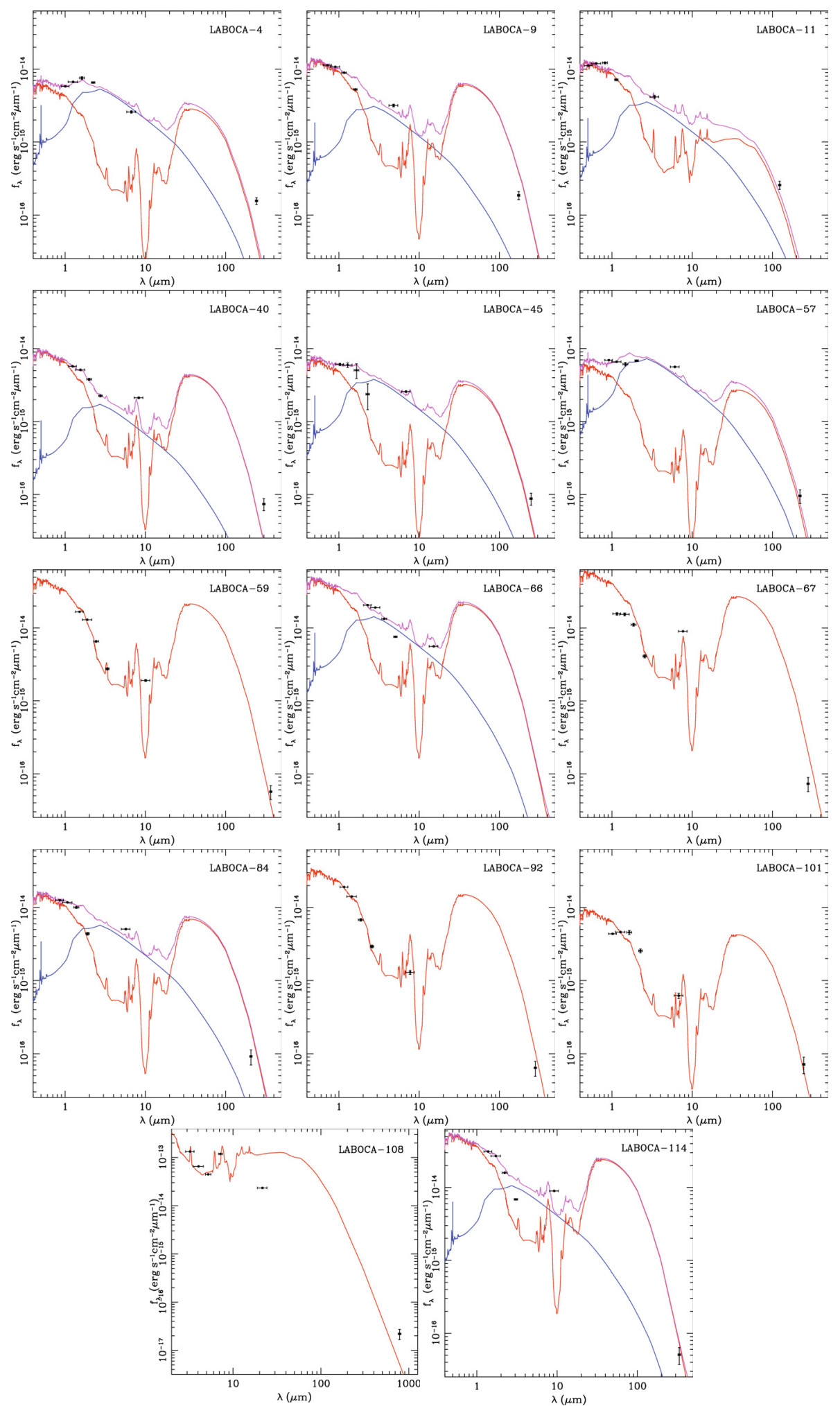
and blue lines represent the star-forming and torus templates of Polletta et al. (2007), while the purple curve denotes the total model.

\section{X-ray stacking analysis}

Having dealt with the properties of the X-ray detected SMGs, we attempt to gain an insight into the average $\mathrm{X}$-ray properties of the individually undetected LABOCA sources. This can be done in a statistical manner simply by co-adding (stacking) the $\mathrm{X}$-ray flux around each SMG. Stacking techniques have been widely used in X-ray astronomy to study the mean properties of source populations selected to have certain well-defined properties, and they are too faint in X-rays to be individually detected (e.g. Nandra et al. 2002; Georgantopoulos et al. 2008). A fixed radius aperture is used to extract and to sum the Xray photons at the positions of the LABOCA sources. Sources that lie close to, or are associated with, an X-ray detection are 
Table 3. Infrared luminosities.

\begin{tabular}{llllllll}
\hline \hline ID & $v L_{v}(6 \mu \mathrm{m})$ & $\begin{array}{l}L_{\mathrm{IR}} \\
(1)\end{array}$ & $\begin{array}{l}L_{\text {torus }} / L_{\mathrm{IR}} \\
(2)\end{array}$ & $\begin{array}{l}\log \left(L_{\mathrm{X}} / L_{\mathrm{IR}}\right) \\
(5)\end{array}$ & $\begin{array}{l}\text { IRAC } \\
(6)\end{array}$ & $\begin{array}{l}\text { Full-SED } \\
(7)\end{array}$ & $\begin{array}{l}\text { Prob. } \\
(8)\end{array}$ \\
\hline $\mathrm{W}-4$ & 1.0 & 1.4 & 0.14 & -2.7 & AGN & Arp 220/Torus & 0.5 \\
$\mathrm{~W}-9$ & 1.7 & 7.8 & 0.04 & -1.9 & SF & Arp 220/Torus & 3.9 \\
$\mathrm{~W}-11$ & 5.4 & 4.7 & 0.21 & -2.8 & $\mathrm{SF}$ & M 82/Torus & 2.1 \\
$\mathrm{~W}-40$ & 0.16 & 0.88 & 0.03 & -2.9 & SF & Arp 220/Torus & 0.4 \\
$\mathrm{~W}-45^{\dagger}$ & 0.69 & 1.4 & 0.09 & -3.6 & SF & Arp 220/Torus & 0.9 \\
$\mathrm{~W}-57$ & 2.0 & 1.9 & 0.18 & -2.4 & AGN & Arp 220/Torus & 0.6 \\
$\mathrm{~W}-59^{\dagger}$ & $0.06^{\star}$ & 1.7 & 0.006 & -4.15 & SF & Arp 220 & 42. \\
$\mathrm{~W}-66$ & 0.07 & 0.23 & 0.05 & -1.98 & SF & Arp 220/Torus & 3.9 \\
$\mathrm{~W}-67^{\dagger}$ & $0.76^{\star}$ & 4.2 & 0.03 & -4.24 & SF & Arp 220 & 47. \\
$\mathrm{~W}-84$ & 1.9 & 5.3 & 0.07 & -2.86 & SF & Arp 220/Torus & 2.7 \\
$\mathrm{~W}-92$ & $0.0^{\star}$ & 3.8 & 0.00 & -3.30 & SF & Arp 220 & 99. \\
$\mathrm{~W}-101$ & $0.22^{\star}$ & 1.30 & 0.03 & -2.92 & SF & Arp 220 & 11.1 \\
$\mathrm{~W}-108^{\dagger}$ & $0.002^{\star}$ & 0.02 & 0.03 & -3.84 & SF & M 82 & 17.1 \\
$\mathrm{~W}-114$ & $0.65^{\star}$ & 3.3 & 0.03 & -4.01 & SF & Arp 220/Torus & 5.2 \\
\hline
\end{tabular}

Notes. ${ }^{(\dagger)}$ Denotes a possible star-forming galaxy on the basis of the X-ray properties; ${ }^{\star}$ ) Means that the best-fit value of the torus luminosities are given although such component is not formally required by F-test. The columns are: (1) LABOCA ID as in Table 1; (2) Torus $6 \mu$ m $v L_{v}$ monochromatic luminosity in units of $10^{45} \mathrm{erg} \mathrm{s}^{-1}$; (3) $10-1000 \mu \mathrm{m}$ Infrared Luminosity in units of $10^{46} \mathrm{erg} \mathrm{s}^{-1}$; (4) Ratio of torus to total 10-1000 $\mu \mathrm{m}$ luminosity; (5) Logarithm of the Ratio of X-ray luminosity to total IR (10-1000 $\mu \mathrm{m}$ ) luminosity; (6) mid-IR IRAC template: powerlaw (AGN) vs. curved (star-forming); (7) Best-fit templates from Polletta et al. (2007); (8) Probability $\left(\times 10^{-2}\right)$ that the torus component is not needed according to the F-test.

Table 4. X-ray stacking results.

\begin{tabular}{lllllll}
\hline \hline $\begin{array}{l}\text { Sample } \\
(1)\end{array}$ & $\begin{array}{l}\text { No. } \\
(2)\end{array}$ & $\begin{array}{l}\text { Soft counts } \\
(3)\end{array}$ & $\begin{array}{l}\text { Soft flux } \\
(4)\end{array}$ & $\begin{array}{l}\text { Hard counts } \\
(5)\end{array}$ & $\begin{array}{l}\text { Hard flux } \\
(6)\end{array}$ & $\begin{array}{l}\text { HR } \\
(7)\end{array}$ \\
\hline LABOCA eCDFS & 100 & $61(5.6 \sigma)$ & $2.3 \times 10^{-17}$ & $26(2.2 \sigma)$ & $2.2 \times 10^{-17}$ & $-0.40 \pm 0.10$ \\
LABOCA CDFS & 21 & $28(2.5 \sigma)$ & $5 \times 10^{-18}$ & $16(1.3 \sigma)$ & $<1.6 \times 10^{-17}$ & $>-0.06^{\dagger}$ \\
\hline
\end{tabular}

Notes. (1), (2) sample where stacking technique has been applied and number of sources; (3) Net soft counts and significance of signal; (4) Soft flux (0.5-2 keV) in units of erg cm $\mathrm{em}^{-2} \mathrm{~s}^{-1}$; (5) Hard net counts and significance; (6) Hard flux (2-5 keV) in units of erg cm ${ }^{-2} \mathrm{~s}^{-1}$; (7) Hardness ratio. (†) The upper limit on flux and the corresponding lower limit on the hardness ratio correspond to $2 \sigma$.

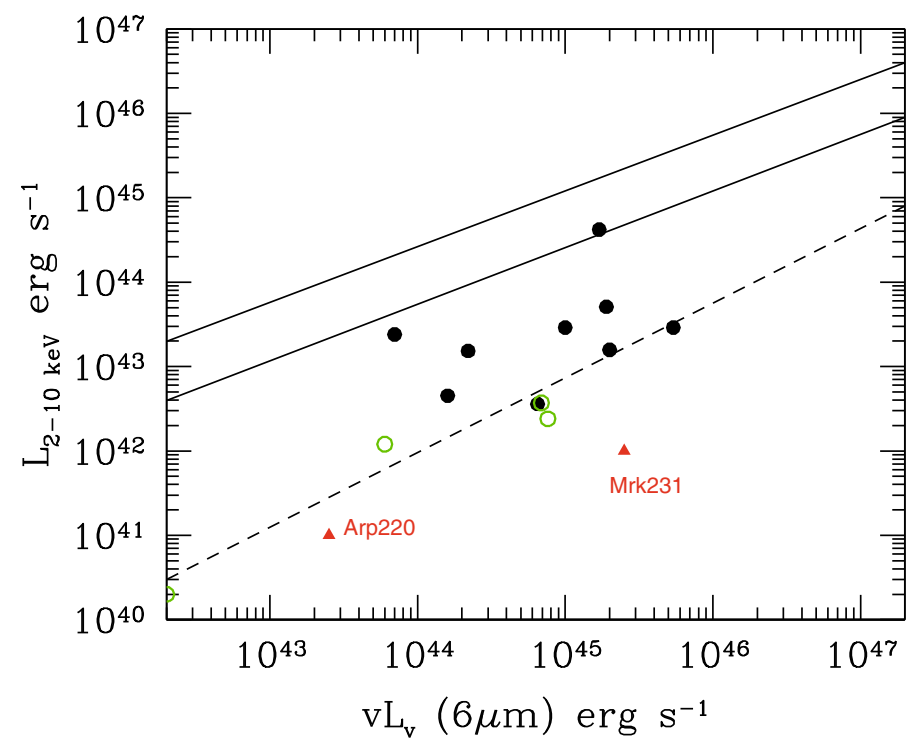

Fig. 6. The X-ray (2-10 keV) luminosity (uncorrected for absorption) as a function of the torus $6 \mu \mathrm{m}$ luminosity. Filled (black) circles represent the AGN while open (green) circles denote the candidate galaxies. Only nine AGN are plotted, because source W-92 has no torus contribution. The solid lines denote the region occupied by unabsorbed AGN, while the region beyond the short-dashed line is populated by Compton-thick AGN in the local Universe (adapted from Alexander et al. 2008). For comparison we plot the positions of Arp 220 and the Compton-thick AGN Mrk231 in the diagram. excluded from the analysis to avoid contamination of the stacked signal from the X-ray photons of detected sources. To maximise the signal we adopt an extraction radius of 3 arcsec around the MIPS $24 \mu \mathrm{m}$ counterparts of the LABOCA sources. A 3 arcsec aperture encloses more than about $90 \%$ of the photons in the soft $0.5-2 \mathrm{keV}$ spectral band at an off-axis angle of 5 arcmin. We perform the stacking analysis in two energy bands: the soft $(0.5-2 \mathrm{keV})$ and the hard $(2-5 \mathrm{keV})$. The choice of the latter band minimises the instrumental background contamination thus maximising the sensitivity of the stacking analysis. We perform the stacking analysis for the LABOCA sources lying in both the eCDFS and CDFS regions.

The significance of the stacked signal depends on the value of the background. This is estimated by using the background maps produced by the WAVDETECT task of CIAO. We sum the X-ray photons in regions around each source used in the stacking. The significance of the stacked signal in background standard deviations is estimated by $(T-B) / T$, where $T$ and $B$ are the total (source + background) and background counts, respectively. Fluxes are determined by multiplying the stacked count rate by the appropriate energy conversion factor, which is estimated separately for each class of sources, based on the spectral shape of the stacked signal. A rough estimate of the mean spectral index is obtained by deriving the hardness ratio $(\mathrm{HR}=(h-s) /(h+s))$ between the $s=0.5-2 \mathrm{keV}$ and $h=2-5 \mathrm{keV}$ bands.

The stacking results are summarised in Table 4 . We see that the stacking of the LABOCA sources in the eCDFS reveals a 


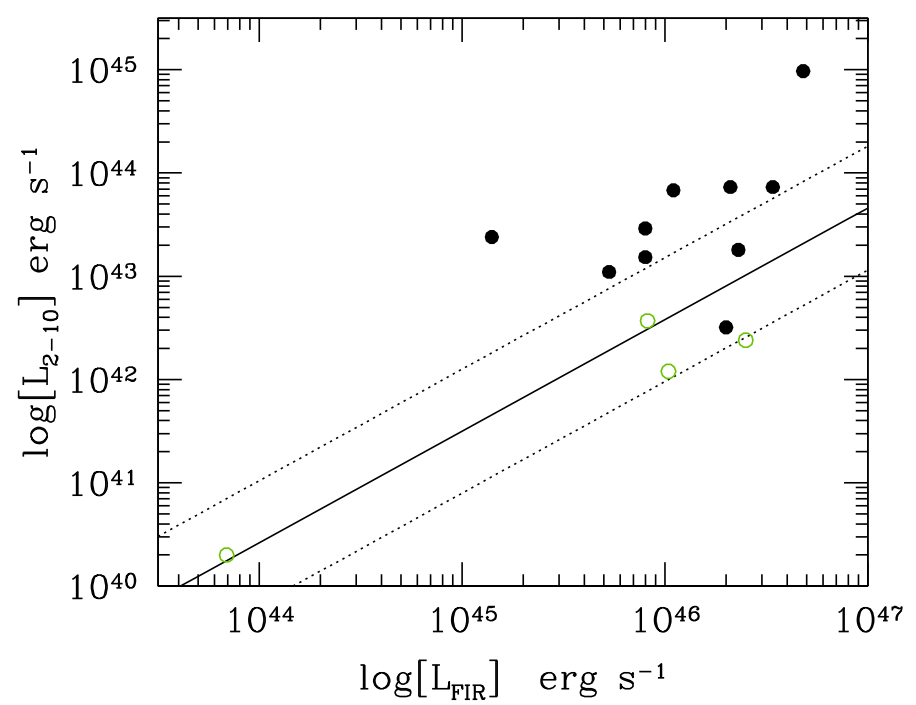

Fig. 7. The X-ray $(2-10 \mathrm{keV})$ vs. the FIR $(4-100 \mu \mathrm{m})$ relation for our sources. The solid and dotted lines denote the best-fit relation of Ranalli et al. (2003), and its associated $2 \sigma$ error, for normal galaxies in the local Universe. Open circles denote the four sources classified as galaxies on the basis of the X-ray diagnostics.

strong signal in the soft band, while the signal is marginal in the hard band. We checked the significance of the soft signal by performing 100 simulations around random positions on the image. There is no simulation where we obtain a signal higher than the actually detected signal (61 counts), implying that the confidence limit for our detection is higher than $99 \%$. The implied flux in the soft band is $2.3 \times 10^{-17} \mathrm{erg} \mathrm{cm}^{-2} \mathrm{~s}^{-1}$, i.e. about four times fainter than the flux limit in the eCDFS (see Virani et al. 2008; Lehmer et al. 2005). We find a hardness ratio of $-0.40 \pm 0.10$ corresponding to a photon index of $\Gamma \approx 1.6$. In the CDFS we barely detect a signal even in the soft band. This is most probably because there are few SMGs employed in the stacking analysis.

\section{Discussion}

In the previous sections we presented a comprehensive analysis of the X-ray and mid-IR properties of the SMG galaxies in the eCDFS and CDFS. These properties are used to assess (1) the fraction of AGN among the SMG population; (2) the amount of obscuration and in particular Compton-thick obscuration; and (3) the power-mechanism among the SMG that host an AGN

\subsection{Fraction of $A G N$ among SMGs}

Using robust positions from Spitzer MIPS, we find 14 associations of X-ray sources with LABOCA SMGs or a fraction of $(11 \pm 3 \%)$. As the observations in the area of the CDFS are very sensitive, it is very likely that a significant number of sources are associated with normal galaxies rather than AGN (see Hornschemeier et al. 2003; Tzanavaris \& Georgantopoulos 2008; Ptak et al. 2008, and references therein). The X-ray analysis has suggested that four SMGs are associated with normal galaxies rather than AGN. This was based on their relatively low X-ray luminosities (less than a few times $10^{42} \mathrm{erg} \mathrm{s}^{-1}$ ) in combination with unobscured X-ray spectra. A further check on whether these sources are associated with normal galaxies can be provided by the $L_{\mathrm{x}}-L_{\mathrm{FIR}}$ relation of Ranalli et al. (2003). These authors have found a strong correlation between the X-ray and the IR luminosity in star-forming galaxies in the local Universe. This implies that only sources with an excess of X-ray emission above this relation can be securely associated with AGN. We show this relation in Fig. 7. It can be seen that the four sources classified as galaxies on the basis of the X-ray diagnostics alone also follow the $L_{\mathrm{X}}-L_{\mathrm{FIR}}$ relation of Ranalli et al. (2003). A fifth source that falls below the Ranalli et al. (2003) relation has a flat spectrum, so it is most probably an AGN (W-114).

Excluding the four candidate galaxies, the fraction of X-ray detected AGN among the LABOCA SMGs amounts to 10/126 $(8 \pm 2) \%$. Constraining this analysis in the CDFs where the sensitivity is the highest yields a higher AGN fraction, as expected. In particular, there are 38 LABOCA sources in CDFS; out of these, ten are associated with X-ray sources translating to a percentage of X-ray detections of $(26 \pm 9) \%$. Because among these ten sources three are most likely associated with galaxies, a conservative estimate for the AGN fraction amounts to $7 / 38(18 \pm 7) \%$.

We compared the figures above with the fraction of X-ray sources and AGN, in particular, that Alexander et al. (2005b) and Laird et al. (2010) have derived in the CDFN. As the X-ray sensitivity of the CDFS and CDFN are identical this comparison is straightforward. Alexander et al. (2005b) find a large number of $\mathrm{X}$-ray detections among radio-detected SMGs $(75 \pm 19 \%)$. Laird et al. (2010) find a lower fraction of X-ray detections among the overall SMG population $(45 \pm 8 \%)$. The primary difference between the analysis of Laird et al. (2010) and that of Alexander et al. (2005b) is that the former uses a purely sub-mm selected sample (Pope et al. 2006), while the latter employs a mixture of SMGs and radio sources with follow-up SCUBA photometry; therefore, the sample of Laird et al. (2010) which contains 35 SMGs is more appropriate for comparison with our work. Although this sample gives a higher fraction of X-ray detections compared with our sample, their AGN fraction of about $25 \pm 8 \%$ reasonably agrees with the results presented here. Finally, Hill \& Shanks (2010) present a cross-correlation of X-ray sources in the eCDFS with LABOCA sources. They estimate that the contribution of X-ray sources to the sub-mm background is only $\approx 3 \%$.

The AGN fraction derived here can only be considered as a lower limit to the true AGN fraction. This is because there may be many more low-luminosity (for example, obscured) AGN among the non-X-ray detected SMGs. The X-ray stacking analysis could shed more light on such a possibility. A strong signal is detected in the soft band in the eCDFS with a corresponding flux of $2 \times 10^{-17} \mathrm{erg} \mathrm{cm}^{-2} \mathrm{~s}^{-1}$. If we adopt the average redshift of the non X-ray detections in the SMG sample of Pope et al. (2006) $(z=2.1)$ as the median redshift of our non X-ray detected SMGs, we find that their X-ray luminosity amounts to $5 \times 10^{41} \mathrm{erg} \mathrm{s}^{-1}$. The luminosity obtained above is comparable to what is obtained by Laird et al. (2010) for their X-ray undetected SMGs in the soft band $\left(4 \times 10^{41} \mathrm{erg} \mathrm{s}^{-1}\right)$. The hardness ratio of $-0.40 \pm 0.10$ shows relatively little absorption. In particular, this hardness ratio corresponds to $N_{\mathrm{H}}=1 \times 10^{21} \mathrm{~cm}^{-2}$ in the observer's frame, assuming a photon index of $\Gamma=1.8$ (or $N_{\mathrm{H}} \approx 2 \times 10^{22} \mathrm{~cm}^{-2}$ at $z=2$ ). Alternatively, this hardness ratio corresponds to a photon-index of $\Gamma \approx 1.6$ (for $N_{\mathrm{H}}=0$ ). Given that the implied spectrum is relatively unobscured, it is reasonable to assume that the bulk of the X-ray emission comes from star-forming processes and hence to estimate the SFR. As the IR luminosity provides a robust measurement of the SFR, the tight $L_{\mathrm{X}}-L_{\mathrm{FIR}}$ correlation found by Ranalli et al. (2003) implies that X-rays are also a good tracer of the star formation in galaxies. Based on their $L_{X}-L_{\mathrm{IR}}-\mathrm{SFR}$ correlation, we find an average SFR of $110 M_{\odot} \mathrm{yr}^{-1}$ for our non-X-ray detected sources. 
This agrees with the value derived by Laird et al. (2010), namely $150 M_{\odot} \mathrm{yr}^{-1}$. As these authors point out, this value is well below the huge SFR $\left(\sim 1000 M_{\odot} \mathrm{yr}^{-1}\right)$ derived for SMGs on the basis of the FIR luminosities. This is also below the SFR derived for the three most luminous candidate galaxies in our sample (W-45, W-59 and W-67), which ranges between $\sim 300-1000 M_{\odot} \mathrm{yr}^{-1}$, according to the X-ray luminosity and the relation of Ranalli et al. (2003). It is rather unlikely that the low SFR derived from the stacked signal in SMGs can be attributed to obscured star formation. For example, if the column density is $N_{\mathrm{H}} \sim 10^{22} \mathrm{~cm}^{-2}$ at $z=2$ as suggested above, the $0.5-2 \mathrm{keV}$ luminosity and thus the SFR would increase by a factor of only 1.5 .

\subsection{Obscuration}

The direct X-ray spectral analysis reveals that the majority of AGN are significantly absorbed with column densities above $10^{23} \mathrm{~cm}^{-2}$ (see Fig. 3). One of the sources could even be classified as a transmission-dominated Compton-thick AGN: W-11 at a photometric resdhift of $z=6.07$. Nevertheless, this classification is highly ambiguous because of the associated redshift uncertainties. The $L_{\mathrm{x}}-L_{6 \mu \mathrm{m}}$ diagram suggests that source W-114 may be related to a reflection-dominated Compton-thick source. Its flat X-ray spectrum $(\Gamma<1.32$ at the $90 \%$ confidence limit $)$ also points towards this interpretation. Our results on the amount of obscuration agree reasonably with those of Laird et al. (2010) in the CDFN. These authors find seven bona-fide AGN among their $18 \mathrm{SMG} / \mathrm{X}$-ray associations. Their X-ray spectral analysis shows a median obscuration of $\sim 10^{23} \mathrm{~cm}^{-2}$ while one of their sources is a Compton-thick AGN.

The stacking analysis provides significant constraints on the fraction of Compton-thick sources among the SMG undetected in X-rays. The detected signal is relatively soft $(\mathrm{hr}=-0.40$ between the $0.5-2 \mathrm{keV}$ and the $2-5 \mathrm{keV}$ bands), implying a spectrum with a photon index of $\Gamma=1.6$. This immediately suggests there are few hard spectrum sources such as those associated with Compton-thick AGN. To quantify the fraction of Comptonthick AGN, we assume a simplistic model where the undetected SMG population consists solely of star-forming galaxies and Compton-thick AGN. We assume that normal galaxies have an average spectrum of $\Gamma=1.7$, (e.g. Zezas et al. 1998) which corresponds to a hardness ratio of -0.44 in the above spectral bands. We then adopt a spectrum of $\Gamma=1$ for Compton-thick sources (e.g. Matt et al. 2004; Georgantopoulos et al. 2009) or equivalently a hardness ratio of -0.13 . We derive a fraction of Compton-thick sources of about $10 \%$. This estimate is only valid under the assumption that the X-ray fluxes from all the sources are roughly the same. This fraction should only be considered as an upper limit. If there are AGN with significant obscuration (but not necessarily Compton-thick) among the undetected SMG sources, the fraction of Compton-thick sources will be lower. For example, AGN with a rest-frame column density of $N_{\mathrm{H}}=10^{23} \mathrm{~cm}^{-2}$ at $z=2$ have a hardness ratio similar to that of a reflection-dominated Compton-thick AGN with $\Gamma=1$.

\subsection{The SMG power mechanism}

We discuss here the possibility that the AGN powers the total IR emission in the case of our ten bona-fide AGN; i.e., we exclude the four sources classified as possible galaxies on the basis of low X-ray luminosities and soft X-ray spectra (see Sect. 8.1 above). The SED fitting provides a clear picture of the AGN contribution. We find that even in the two sources that have

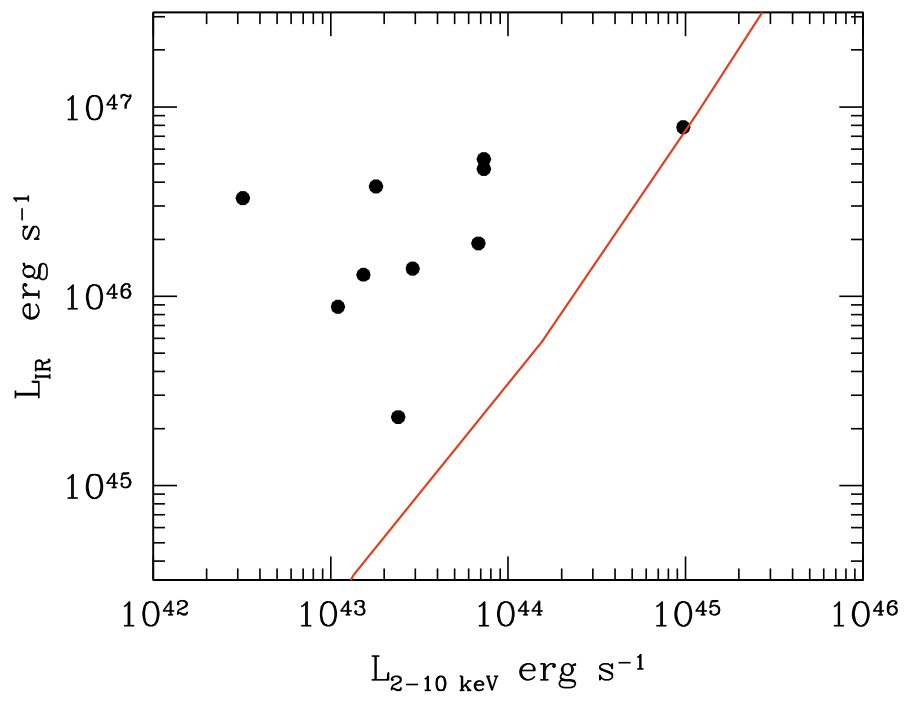

Fig. 8. The X-ray $(2-10 \mathrm{keV})$ vs. the total IR $(10-1000 \mu \mathrm{m})$ luminosity relation for our sources. The solid line denotes the empirical relation of Hopkins et al. (2007) for the bolometric correction of AGN.

power-law IRAC photometric distributions and thus are clearcut AGN in the mid-IR part of the spectrum, the torus contributes less than $20 \%$ of the total IR $(10-1000 \mu \mathrm{m})$ luminosity. Laird et al. (2010) estimate a fraction of 3/16 for AGN-powered SMGs in their sample.

In order to visualize better the level of the AGN contribution, we plot the total IR luminosity as a function of the X-ray luminosity in Fig. 8 for our sources, together with the empirically estimated relation for AGN (Hopkins et al. 2007). We see that only one source (W-9) follows this relation, while the remaining sources are far above the relation of Hopkins et al. (2007), implying a negligible AGN contribution.

\section{Conclusions}

We explore the X-ray properties of the 126 sub-mm sources of the LABOCA survey in the area of the CDFS and eCDFS. Using Spitzer and radio counterparts of the sub-mm sources where available, we find 14 sources with significant X-ray detection. Our results can be summarised as:

- The X-ray luminosities and spectra suggest that most of the sub-mm - X-rays associations (10/14) host an AGN. In four sources the X-ray emission could possibly develop out of star-forming processes.

- The fraction of X-ray AGN among the LABOCA SMG sample in the area of the CDF-S is at most $26 \pm 9 \%$.

- Six of our X-ray sources show significant amounts of absorption $N_{\mathrm{H}}>10^{23} \mathrm{~cm}^{-2}$ but there is no unambiguous evidence that any of the sources is a Compton-thick AGN.

- Detailed SED fitting shows that most of the AGN require a torus component. However, the AGN contribution to the total IR luminosity is small.

- X-ray stacking analysis of the undetected SMGs reveals a signal with a relatively soft spectrum. This is more suggestive of a SFR galaxy population.

Acknowledgements. I.G. and A.C. acknowledge the Marie Curie fellowship FP7-PEOPLE-IEF-2008 Prop. 235285. A.C. acknowledges receipt of ASI grants $I / 023 / 05 / 00$ and $I / 88 / 06$. This publication is based on data acquired with the Atacama Pathnder Experiment (APEX) under programme numbers 078.F-9028(A), 079.F-9500(A), 080.A-3023(A), and 081.F-9500(A). APEX is a collaboration between the Max-Planck-Institut fur Radioastronomie, the 
European Southern Observatory, and the Onsala Space Observatory. We acknowledge the use of Spitzer data provided by the Spitzer Science Center. The Chandra data were taken from the Chandra Data Archive at the Chandra X-ray Center.

\section{References}

Alexander, D. M., Bauer, F. E., Brandt, W. N., et al. 2003, AJ, 126, 539 Alexander, D. M., Smail, I., Bauer, F. E., \& Chapman, S. C. 2005a, Nature, 434, 738

Alexander, D. M., Bauer, F. E., Chapman, S. C., et al. 2005b, ApJ, 632, 736

Alexander, D. M., Chary, R. R., Pope, A., et al. 2008, ApJ, 687, 835

Alonso-Herrero, A., Pérez-González, P. G., Alexander, D. M., et al. 2006, ApJ, 640,127

Aretxaga, I., Hughes, D. H., Coppin, K., et al. 2007, MNRAS, 376, 1571

Armus, L., Charmandaris, V., Bernard-Salas, J., et al. 2007, ApJ, 656, 148

Arnaud, K. A. 1996, ASP Conf. Ser., 101, 17

Barger, A. J., Cowie, L. L., Sanders, D. B., et al. 1998, Nature, 394, 248

Bauer, F. E., Yan, L., Sajina, A., \& Alexander, D. M. 2010, ApJ, 710, 212

Bertin, E., \& Arnouts, S. 1996, A\&AS, 117, 393

Blain, A. W., Smail, I., Ivison, R. J., \& Kneib, J.-P. 1999, MNRAS, 302, 632

Blain, A. W., Smail, I., Ivison, R. J., Kneib, J.-P., \& Frayer, D. T. 2002, PhR, 369,111

Braito, V., Della Ceca, R., Piconcelli, E., et al. 2004, A\&A, 420, 79

Brammer, G. P., van Dokkum, P. G., \& Coppin, P. 2008, ApJ, 686, 1503

Capak, P., Carilli, C. L., Lee, N., et al. 2008, ApL, 681, L53

Cash, W. 1979, ApJ, 228, 939

Chapin, E. L., Chapman, S. C., Coppin, E., et al. 2010, MNRAS, in press

Chapman, S. C., Blain, A. W., Smail, I., \& Ivison, R. J. 2003

Chapman, S. C., Blain, A. W., Smail, I., \& Ivison, R. J. 2005, ApJ, 622, 772

Coppin, K. E. K., Smail, I., Alexander, D. M., et al. 2009, MNRAS, 395, 1905

Daddi, E., Dannerbauer, H., Stern, D., et al. 2009, ApJ, 694, 1517

Dickey, J. M., \& Lockman, F. J. 1990, ARA\&A, 28, 215

Dickinson, M., Giavalisco, M., \& The Goods Team 2003, in The Mass of Galaxies at Low and High Redshift, ed. R. Bender, \& A. Renzini (Springer-Verlag), 324

Dickinson, M., Alexander, D., Bell, E., et al. 2006, Sptz. Prop, 30948

Donley, J. L., Rieke, G. H., Pérez-González, P. G., \& Barro, G. 2008, ApJ, 687, 111

Gawiser, E., van Dokkum, P. G., Herrera, D., et al. 2006, ApJS, 162, 1

Georgakakis, A., Georgantopoulos, I., Akylas, A., Zezas, A., \& Tzanavaris, P. 2006, ApJ, 641, L101

Georgakakis, A., Rowan-Robinson, M., Babbedge, T. S. R., \& Georgantopoulos, I. 2007, MNRAS, 377, 203

Georgantopoulos, I., Georgakakis, A., Rowan-Robinson, M., \& Rovilos, E. 2008, A\&A, 484, 671

Georgantopoulos, I., Akylas, A., Georgakakis, A., \& Rowan-Robinson, M. 2009, A\&A, 507, 747

Giacconi, R., Zirm, A., Wang, J., et al. 2002, ApJS, 139, 369

Giavalisco, M., Ferguson, H. C., Koekemoer, A. M., et al. 2004, ApJ, 600, 93

Grazian, A., Fontana, A., de Santis, C., et al. 2006, A\&A, 449, 951

Güsten, R., Nyman, L. A., Schilke, P., et al. 2006, A\&A, 454, L13
Hill, M. D., \& Shanks, T. 2010, MNRAS, in press

Hopkins, P. F., Richards, G. T., \& Hernquist, L. 2007, ApJ, 654, 731

Hornschemeier, A. E., Bauer, F. E., Alexander, D. M., et al. 2003, AJ, 126, 575

Hughes, D. H., Serjeant, S., Dunlop, J., et al. 1998, Nature, 394, 241

Iwasawa, K., Sanders, D. B., Evans, A. S., et al. 2005, MNRAS, 357, 565

Kellermann, K. I., Fomalont, E. B., Mainieri, V., et al. 2008, ApJS, 179, 71

Kriek, M., van Dokkum, P. G., Franx, M., et al. 2006, ApJ, 649, L71

Laird, E. S., Nandra, K., Pope, A., \& Scott, D. 2010, MNRAS, 401, 2763

Lehmer, B. D., Brandt, W. N., Alexander, D. M., et al. 2005, ApJS, 161, 21

Luo, B., Bauer, F. E., Brandt, W. N., et al. 2008, ApJS, 179, 19

Luo, B., Brandt, W. N., Xue, Y. Q., et al. 2010, ApJS, 187, 560

Lutz, D., Maiolino, R., Spoon, H. W. W., \& Moorwood, A. F. M. 2004, A\&A, 418,465

Lutz, D., Mainieri, V., Rafferty, D., et al. 2010, ApJ, 712, 1287

Magnelli, B., Elbaz, D., Chary, R. R., et al. 2009, A\&A, 496, 57

Maiolino, R. 2008, NewA, 52, 339

Matt, G., Bianchi, S., Guainazzi, M., \& Molendi, S. 2004, A\&A, 421, 473

Menéndez-Delmestre, K., Blain, A. W., Alexander, D. M., et al. 2007, ApJ, 655, L65

Menéndez-Delmestre, K., Blain, A. W., Smail, I., et al. 2009, ApJ, 699, 667

Moran, E. C., Lehnert, M. D., \& Helfand, D. J. 1998, ApJ, 526, 649

Nandra, K., \& Pounds, K. 1994, MNRAS, 268, 405

Nandra, K., Mushotzky, R. F., Arnaud, K., et al. 2002, ApJ, 576, 625

Polletta, M., Wilkes, B. J., Siana, B., et al. 2006, ApJ, 642, 673

Polletta, M., Tajer, M., Maraschi, L., et al. 2007, ApJ, 663, 81

Pope, A., Scott, D., Dickinson, M., et al. 2006, MNRAS, 370, 1185

Pope, A., Chary, R.-R., \& Alexander, D. M. 2008, ApJ, 675, 1171

Ptak, A., Mobasher, B., Hornschemeier, A., Bauer, F., \& Norman, C. 2007, ApJ, 667,826

Ranalli, P., Comastri, A., \& Setti, G. 2003, A\&A, 399, 39

Rovilos, E., Georgantopoulos, I., Tzanavaris, P., et al. 2009, A\&A, 502, 85

Rovilos, E., Georgantopoulos, I., Akylas, A., \& Fotopoulou, S. 2010, A\&A, 522, A11

Rix, H.-W., Barden, M., Beckwith, S. V. W., et al. 2004, ApJS, 152, 163

Shao, L., Lutz, D., Nordon, R., et al. 2010 A\&A, 518, L26

Shioya, Y., Taniguchi, Y., \& Trentham, N. 2001, MNRAS, 321, 11

Siringo, G., Kreysa, E., Kovács, A., et al. 2009, A\&A, 497, 945

Smail, I., Ivison, R. J., \& Blain, A. W. 1997, ApJ, 490, L5

Sutherland, W., \& Saunders, W. 1992, MNRAS, 259, 413

Szokoly, G. P., Bergeron, J., Hasinger, G., et al. 2004, ApJS, 155, 271

Taylor, E. N., Franx, M., van Dokkum, P. G., et al. 2009, ApJS, 183, 295

Tozzi, P., Gilli, R., Mainieri, V., et al. 2006, A\&A, 451, 457

Tzanavaris, P., \& Georgantopoulos, I. 2008, A\&A, 480, 663

Tzanavaris, P., Georgantopoulos, I., \& Georgakakis, A. 2006, A\&A, 454, 447

Valiante, E., Lutz, D., Sturm, E., et al. 2007, ApJ, 660, 1060

Virani, S. N., Treister, E., Urry, C. M., \& Gawiser, E. 2006, AJ, 131, 2373

Wall, J. V., Pope, A., \& Scott, D. 2008, MNRAS, 383, 435

Wardlow, J. L., Smail, I., Coppin, K. E. K., et al. 2010, MNRAS, submitted [arXiv: 1006.2137]

Weiß, A., Kovács, A., Coppin, K., et al. 2009, ApJ, 707, 1201

Werner, M. W. 2000, SPIE, 4131, 1

Zezas, A. L., Georgantopoulos, I., \& Ward, M. J. 1998, MNRAS, 301, 915 